\title{
NUMERICAL SIMULATION OF SPRAY BREAK-UP FROM CAVITATING NOZZLE FLOW BY COMBINED EULERIAN- EULERIAN AND VOLUME-OF-FLUID METHODS
}

\author{
WILFRIED EDELBAUER ${ }^{1}$, PATRICK KOLAR ${ }^{1}$, DAVID SCHELLANDER ${ }^{1}$, \\ ZORAN PAVLOVIC ${ }^{2}, \&$ RAIMUND ALMBAUER ${ }^{3}$ \\ ${ }^{1}$ Advanced Simulation Technologies, AVL List GmbH, Graz, Austria. \\ ${ }^{2}$ Advanced Simulation Technologies, AVL List GmbH, Maribor, Slovenia. \\ ${ }^{3}$ Institute of Internal Combustion Engines and Thermodynamics, TU Graz, Austria.
}

\begin{abstract}
The present study shows new results from the recently proposed method for numerical simulations of the spray break-up of cavitating liquid jets. A three-component system consisting of liquid, vapor and gas is applied for the volume-of-fluid simulation of the liquid disintegration in order to track the liquid-gas interface. To keep the numerical effort moderate, the liquid-vapor interface is not resolved by the computational grid, there mass and momentum transfer are described within the Eulerian-Eulerian framework. The numerical method is applied on a simplified injector-like geometry from the literature operated with gasoline at low pressure difference. For quantification of the detached spray ligaments, a new evaluation algorithm has been developed and implemented into the applied CFD code. It scans the liquid volume fraction field for separated ligaments, and determines their position, size and velocity. Additionally the ligament extensions along the principal axes of inertia are determined in order to evaluate the non-sphericity of each ligament after break-up. The presented simulation technique allows detailed numerical investigations of the spray formation process on the micro-scale by taking into account nozzle cavitation, turbulence and aerodynamic forces.

Keywords: cavitation, droplet size distribution, liquid disintegration, non-spherical droplets, spray break-up, surface-tracking, volume-of-fluid
\end{abstract}

\section{INTRODUCTION}

The liquid disintegration during the spray formation process is mainly driven by three effects: nozzle turbulence, aerodynamic interaction and when the minimum pressure falls below the saturation pressure, cavitation. While the first two effects are commonly described in numerical simulations with surface-capturing methods, e.g. [1-3] and several others, the latter effect - cavitation - is often omitted. The reason for this can be found in the increased physical modelling effort. Beside of the phases for liquid and ambient gas, there is need for describing the vapor phase. Furthermore, the vapor bubbles produced by cavitation are in general smaller than the liquid ligaments produced by the primary atomization process. Surface-capturing methods for resolving every single vapor bubble would be computationally too expensive. The method presented here overcomes this problem by modelling the liquid-vapor interface within the Eulerian-Eulerian framework, while the liquid-gas interface is described by the volumeof-fluid (VOF) method.

Only few numerical studies of the liquid break-up with surface-capturing methods by taking into account cavitating nozzle flow have been published in the literature. A pioneer work is reported by Ishimoto et al. [4], who applied VOF and Large Eddy Simulation (LES) for a simplified industrial gasoline injector with cavitation in the nozzle. In this work the three phases, liquid, vapor and gas, are treated like two phases by considering liquid-vapor as cavitating mixture. The authors simulated the primary break-up for a simple nozzle segment with diameter $D=226 \mu \mathrm{m}$. The inlet pressure was $0.444 \mathrm{MPa}$, and the extension of the simulated spray domain was approximately $9 D$. The homogeneous equilibrium model (HEM) 
described by Schmidt et al. [5] was used as cavitation model. Another VOF-LES simulation for cavitation and atomization in gasoline injectors with three phases is reported by Lu et al. [6]. Also there liquid and vapor are treated as compressible single-fluid mixture. Cavitation was modelled by the Sauer-Schnerr model [7]. A 1/6-sector of the complete injector geometry has been simulated, the external domain extension was $5 D$. The same group published a constitutive work where they additionally modelled diffusion between vapor and gas species, as reported by Cailloux et al. [8]. Yu et al. [9] performed numerical studies to investigate the effect of different cavitation models on the spray break-up during the initial stage of injection. The authors applied a three-phase VOF model in a high-pressure diesel injection.

The current paper presents a new application of our recently proposed method [10] for combination of the Eulerian-Eulerian (EE) and the VOF methods in spray simulations with cavitating injector flow. The model deals with three phases, and the liquid-vapor interaction in terms of mass transfer and momentum drag is modelled within the EE framework. This means that the liquid-vapor interface is not resolved by the numerical discretization. The cavitation model is based on the simplified Rayleigh-Plesset equation, and the vapor phase has its own velocity field. The liquid-gas interface is resolved by applying the VOF method. While in Ref. [10] the model was validated on the well-documented water nozzle experiments from Sou et al. [11], and Bicer and Sou [12], the current work shows the simulation of the simplified low-pressure gasoline injector reported by Ishimoto et al. [4]. The presented simulation is performed on a finer computational grid, since the highest mesh resolution is $D / 72$ compared to $D / 56$ in Ref. [4]. This leads to five times more computational cells. Furthermore, a new ligament evaluation algorithm for quantifying non-spherical ligaments is presented.

The paper is organized as follows. After the introduction in the first section, there follows a brief description of the mathematical model including balance equations and the algorithm for ligament detection in the second section. The third sections deals with the numerical simulation set-up and shows the simulation results. The summary and conclusions can be found in the fourth section. All simulations have been performed with the CFD code AVL-FIRE ${ }^{\mathrm{TM}}$ [13], where the presented models have been implemented by the authors.

\section{MATHEMATICAL MODEL}

\subsection{Combination of Eulerian-Eulerian and Volume-of-fluid methods}

Three phases, liquid, vapor and ambient gas, are required for the intended CFD simulation of cavitating nozzle flow and spray break-up. It is assumed that the vapor bubbles produced by cavitation exist on smaller length scales than the liquid ligaments created by spray break-up. As illustrated in Fig. 1, the main idea is to resolve the liquid-gas interface by applying the VOF method between these two phases, but to use the EE approach for modelling the interface between the liquid and the vapor. In Ref. [10] it was shown that the volume fraction and momentum equations for EE and VOF are similar, although the volume fraction and the interphase exchange terms haves different meanings. While for the EE transport equation the volume fraction indicates the ensemble-averaged phase indicator function and is proportional to the amount of the dispersed phase, the VOF volume fraction indicates the location of the fluid-fluid interface, as described by the following equation for phase $k$ :

$$
\alpha_{k}^{V O F}=\left\{\begin{array}{cc}
1 & \text { fully occupied by tracked phase } \\
0 & \text { not occupied by tracked phase } \\
0<\alpha_{k}<1 & \text { interface }
\end{array}\right.
$$




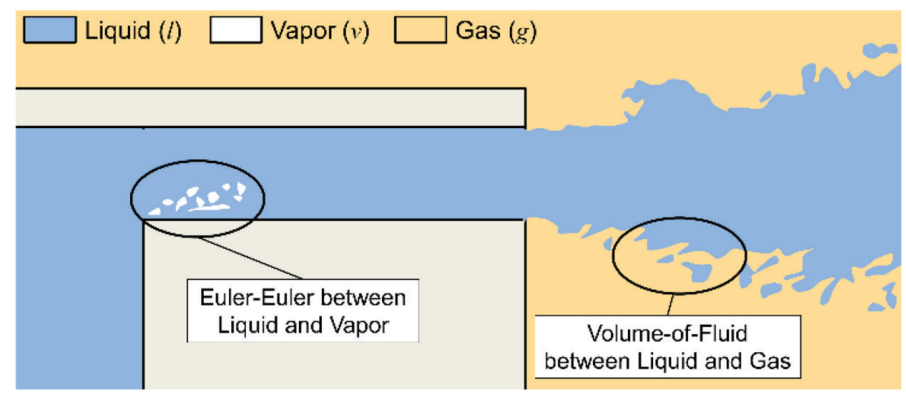

Figure 1: Concept for three-phases simulation by combination of Eulerian-Eulerian and Volume-of-Fluid approaches.

The volume fraction transport equation of the vapor phase, marked by index $v$, is solved according to equation

$$
\frac{\partial \alpha_{v} \rho_{v}}{\partial t}+\frac{\partial}{\partial x_{j}}\left(\alpha_{v} \rho_{v} U_{v, j}\right)=\Gamma_{v l},
$$

where $\rho$ is the density, $U$ is the velocity component and $\Gamma_{v l}$ is the inter-phase mass transfer from cavitation modelled within the EE framework. For the liquid and the gaseous phases, indicated by $k=l$ and $k=g$, respectively, the volume fraction transport is expressed by

$$
\frac{\partial \alpha_{k}}{\partial t}+\frac{\partial}{\partial x_{j}}\left(\alpha_{k} U_{m, j}\right)=S_{\alpha},
$$

where $S_{\alpha}$ is the source/sink which is zero for the gas phase and $-\Gamma_{v l} l \rho_{l}$ for the liquid phase. $U_{m}$ is the mixture velocity, since liquid and gas share the same velocity field. For the momentum equations the considerations are similar to them of the volume fraction equations. A drag model describes the momentum interaction between liquid and vapor within the EE framework, while the surface tension force acts at the VOF-interface between liquid and gas when the equation for the mixture velocity $U_{m}$ is solved. Further details can be found in our previous publication [10].

As cavitation model, the so called 'linear cavitation model' is applied. It is a standard model of the applied CFD code [13] and based on the simplified Rayleigh-Plesset equation for single bubble growth. The model provides a correlation for the mass transfer within the EE framework, as discussed and validated by Alajbegovic et al. $[14]$ and others $[15,16]$. The turbulence is modelled through a LES. The applied model was proposed by Kobayashi et al. $[17,18]$, and is called Coherent Structure Model (CSM). The LES-CSM model uses a local turbulence coefficient, which is based on a coherent structure function. The model is robust, it does not need a wall damping function or additional transport equations, and it can be used for laminar flow.

The aim of the VOF approach is to track the liquid-gas interface. Therefore, special attention is paid on the differencing scheme of the volume fraction eqn (3). It has to ensure that the advected volume fraction field is bounded, monotonic and it has to maintain the sharpness

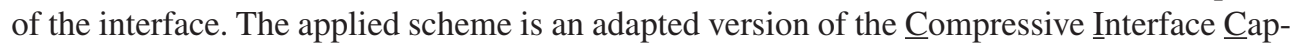
turing $\underline{S}$ cheme for Arbitrary Meshes (CICSAM), which was originally developed by Ubbink 
and Issa [19]. It is based on the donor-acceptor concept by using high resolution schemes. No explicit geometric reconstruction of the interface is employed.

\subsection{Ligament analysis and form diagram}

The droplet size distribution after spray break-up is of particular interest for such kind of simulations. For the determination of droplet or ligament sizes an evaluation algorithm is applied. At selected time instants this feature scans the volume fraction field for separated ligaments, and calculates for each of them the ligament properties, such as center of gravity, volume-equivalent diameter, mean velocity, mass, volume and the ligament surface, as illustrated in Fig. 2 for seven pre-defined ellipsoids. This information is stored in separate output files, which are then used for the evaluation of the mean diameters, i.e. the Sauter mean diameter $D_{32}$, and the droplet size distribution function.

In general the ligaments are non-spherical and have arbitrary shape. Only at the end of the disintegration process, when the droplets are small enough, more or less spherical droplets can be expected. In order to quantify non-spherical ligaments a new method is proposed here. Therefore, the principal axes of inertia are calculated for each individual ligament, and the extensions along these axes, illustrated as $l_{x}, l_{y}$ and $l_{z}$ in Fig. 2 , are determined for the frozen ligament shape at a certain time instant.

Equation (4) describes the moment of inertia tensor with respect to an arbitrary coordinate system $\xi, \eta, \zeta$ and point $A$ as point of origin according to the sketch in Fig. 3 (left). This coordinate system usually represents the coordinates of the computational mesh in the CFD simulation. With Steiner's theorem the moment of inertia with respect to the center of gravity $S, \mathbf{I}_{x y z 0}$ can be calculated according to eqn (5), where $m$ is the ligament mass and $a, b$ and $c$ are the components of the distance vector $\overline{A S}$. The principal axes for inertia, $x_{1}, y_{1}$ and $z_{1}$ are then obtained by solving the Eigenvector problem defined in eqn (6). Since tensor $\mathbf{I}_{x y z 0}$ is symmetric, there exist three real Eigenvalues $\lambda$ obtained from solving the equation $\operatorname{det}\left(\mathbf{I}_{x y z 0}-\lambda \mathbf{E}\right)$, where $\mathbf{E}$ is the unity tensor. The three Eigenvectors are identical to the principal axes of inertia, the Eigenvalues represent the principal moments of inertia.

Once the principal axes of inertia are determined, the extensions of each ligament along these directions, $\tilde{l}_{x}, \tilde{l}_{y}$ and $\tilde{l}_{z}$, are evaluated. By resorting the lengths so that $l_{z} \geq l_{y} \geq l_{x}$ is fulfilled, calculating the ratios $l_{y} / l_{x}$ and $l_{z} / l_{x}$, and drawing them into a diagram, one

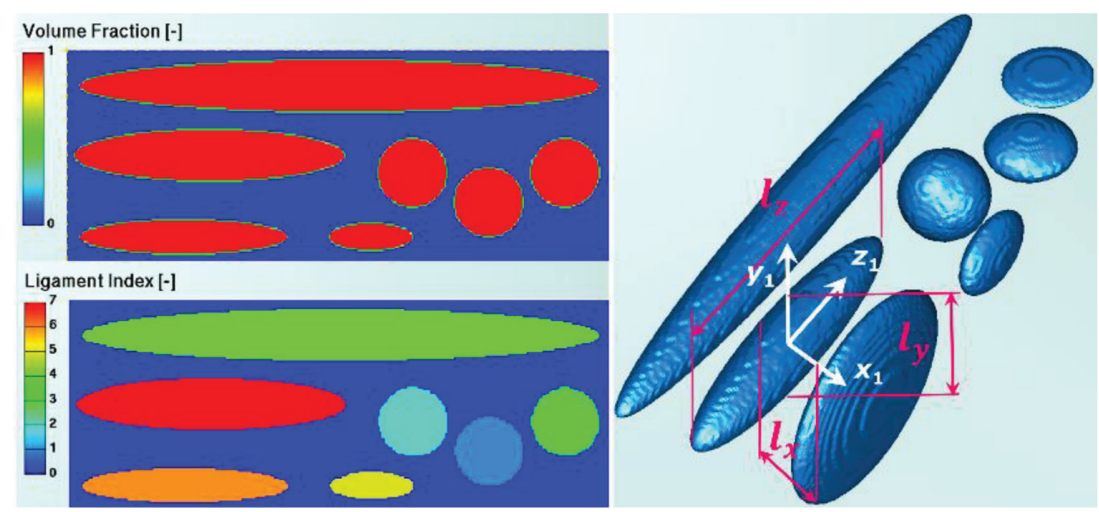

Figure 2: Ligament analysis from the volume fraction field of seven pre-defined ellipsoids. 

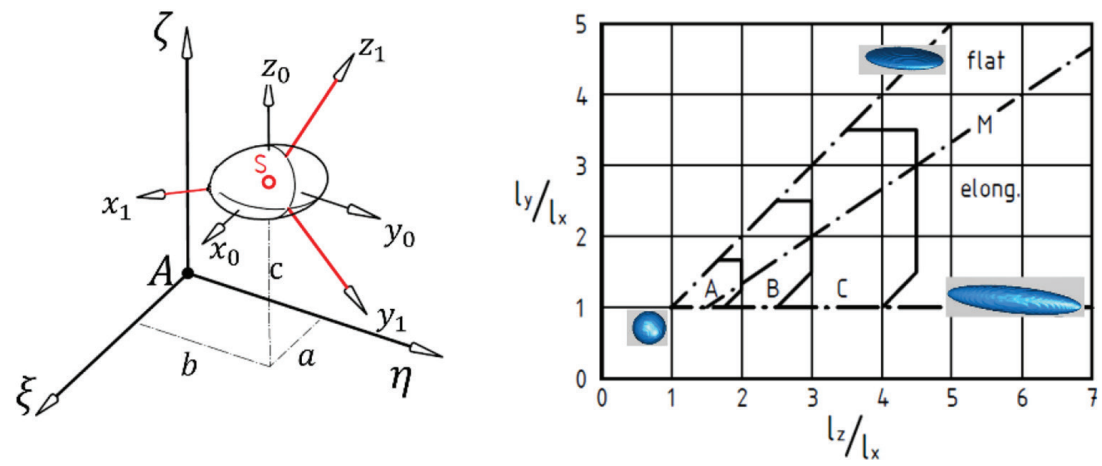

Figure 3: Coordinate system of the ligament (left) and form diagram from Walz [20] (right).

obtains the so-called form diagram proposed by Walz [20], as illustrated in Fig. 3 (right). Ligaments around point (1|1) have spherical shape, elongated or cigar shaped ligaments are located along the horizontal line $l_{y} / l_{x}=1$, and disc shaped ligaments are located along the straight line described by $l_{y} / l_{x}=l_{z} / l_{x}$. All other ligaments can be found in between these borders. The form diagram is a useful illustration for the shape of the droplet size distribution.

$$
\begin{gathered}
\mathbf{I}_{\xi \eta \zeta}=\left[\begin{array}{ccc}
I_{\xi} & I_{\eta \zeta} & I_{\xi \zeta} \\
I_{\eta \zeta} & I_{\eta} & I_{\xi \eta} \\
I_{\xi \zeta} & I_{\xi \eta} & I_{\zeta}
\end{array}\right]=\left[\begin{array}{ccc}
\eta^{2}+\zeta^{2} & \eta \zeta & \xi \zeta \\
\eta \zeta & \xi^{2}+\zeta^{2} & \xi \eta \\
\xi \zeta & \xi \eta & \xi^{2}+\eta^{2}
\end{array}\right] d m . \\
\mathbf{I}_{x y z 0}=\mathbf{I}_{\xi \eta \zeta}-m\left[\begin{array}{ccc}
b^{2}+c^{2} & a b & c a \\
a b & a^{2}+c^{2} & b c \\
c a & b c & a^{2}+b^{2}
\end{array}\right] . \\
\left(\mathbf{I}_{x y z 0}-\lambda_{1, i} \mathbf{E}\right) \mathbf{x}_{1, i}=0 .
\end{gathered}
$$

\section{NUMERICAL SIMULATION}

\subsection{Geometrical model and simulation set-up}

The simulated case is based on the publication of Ishimoto et al. [4] showing a simplified gasoline injector with a short-length nozzle of $L=60 \mu \mathrm{m}$ and $D=226 \mu \mathrm{m}$. Three phases have been defined, and the fluid properties are listed in Table 1. All phases are assumed to be incompressible and isothermal. The injection pressure is $0.444 \mathrm{MPa}$ against $0.1 \mathrm{MPa}$ ambient pressure. This leads to a cavitation number of 0.27 , calculated according to equation $\sigma_{a}=\left(p_{a m b}-p_{s a t}\right) /\left(0.5 \rho_{l} U_{l}^{2}\right)$. The nozzle Reynolds number is 11500 so that turbulent break-up can be expected. The computational mesh showing injector and spray domains, the locations of the boundary conditions and the dimensions is shown in Fig. 4 (left). Inlet and outlet pressure boundaries are located notably far away from the nozzle. At the nozzle wall and the upper side of the spray domain no-slip wall boundary conditions are set. In order to keep the total number of cells moderate, several refinement regions have been applied, as 
Table 1: Fluid properties.

\begin{tabular}{llll}
\hline Fluid & Liquid & Vapor & Air \\
\hline Density $\left[\mathrm{kg} / \mathrm{m}^{3}\right]$ & 732 & 4.62 & 1.134 \\
Dynamic viscosity $[\mathrm{Pa} \cdot \mathrm{s}]$ & $0.442 \cdot 10^{-3}$ & $6.2 \cdot 10^{-6}$ & $1.51 \cdot 10^{-5}$ \\
Surface tension $[\mathrm{N} / \mathrm{m}]$ & 0.0198 & & \\
Saturation pressure $[\mathrm{Pa}]$ & 6155 & & \\
\hline
\end{tabular}

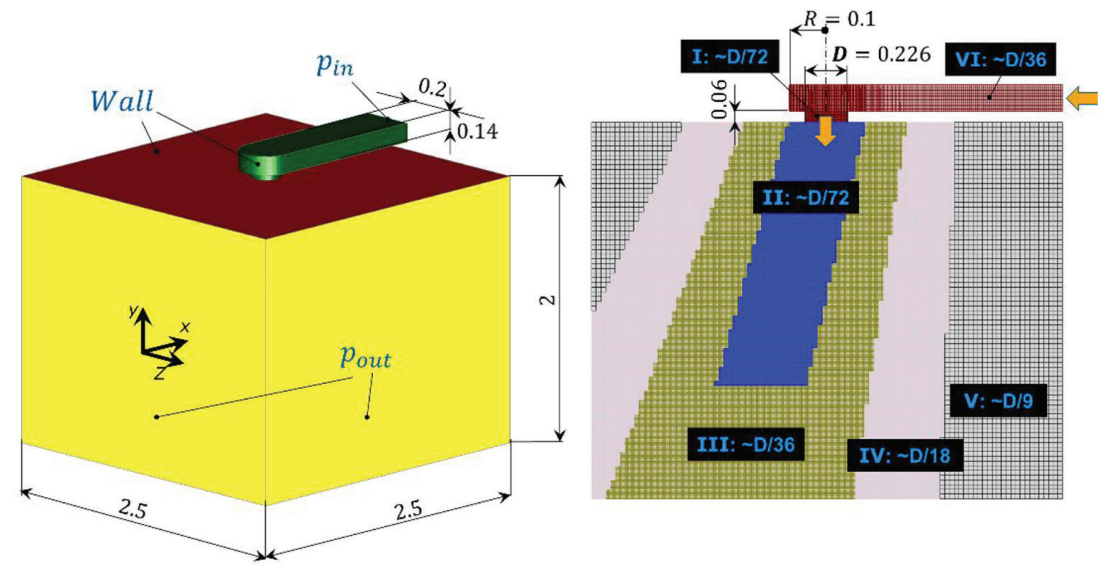

Figure 4: Computational mesh with refinement regions and applied boundary conditions. All dimensions in $\mathrm{mm}$.

shown in Fig. 4 (right). The smallest cells have a size of $3.125 \mu \mathrm{m}$ and are located in injector region I and inner spray region II. Relative to the nozzle diameter this corresponds to a resolution of $D / 72$. Towards the outlet pressure boundaries four refinement levels shaped as interlacing cones from region II to V have been created. Primary break-up is assumed to be finished within the regions with the highest mesh resolution, i.e. regions II and III. The total number of cells is 16.3 million.

The balance equations for volume fraction, velocity and continuity were solved numerically by using the CFD code AVL FIRE ${ }^{\mathrm{TM}}$ which applies a cell-centered finite volume approach. The cell gradients were calculated by using a linear least-square approach. The convection is solved by a variety of differencing schemes, namely SMART-bounded [13, 21] for the momentum equation, central differencing (CD) for the continuity equation, CICSAM for the volume fraction equations of liquid and gas, and upwind for the volume fraction equation of the vapor phase. The time derivative in the balance equations are discretized by the second-order accurate Crank-Nicolson method for the volume fraction equations of liquid and gas, and by the implicit first-order accurate two-level Euler scheme for the other equations. Coupling between pressure and velocity is performed by the segregated SIMPLE-like algorithm, as discussed in Ref. [22, 23] and others. The pressure is assumed to be equal for all phases. An automatic time step algorithm ensures that the CFL number does not exceed the value of 0.4. At initialization the flow field stays at rest, the pressure field is obtained from 
the solution of the potential flow equation. The nozzle is initialized with pure liquid, the spray domain with air.

\subsection{Simulation results}

This section shows selected results from the simulation. A physical time of $1 \mathrm{~ms}$ has been simulated on a Linux cluster (Intel ${ }^{\circledR}$ Xeon ${ }^{\circledR}$ CPU E5-2670 0 @ 2.6 GHz) with 90 cores. The elapsed wall-clock time was 16 days. Figure 5 shows instantaneous flow field results for liquid volume fraction, velocity and pressure in a center plane cut through the nozzle and injector axes at $1 \mathrm{~ms}$. In the volume fraction plot, it can be seen that the liquid-air interface is sharp, and that the length of the liquid core is short. After the distance of approximately three to six times the nozzle diameter, the liquid core is fully disintegrated. Air is sucked back into the nozzle hole and reaches the upper right edge of the nozzle entrance. This creates an asymmetric inflow and leads to the obvious spray deflection from the vertical nozzle axis. Since the nozzle length is very short, $L / D$ is only 0.26 , the deflected flow direction reaches the nozzle exit and causes the observed spray pattern. The velocity plot in Fig. 5 shows a partially turbulent flow field in the nozzle. While the inflow into the injector is laminar, the transition to turbulent flow takes place downstream the edge of the nozzle entrance where an unstable shear layer is formed. Furthermore, there is a dead volume in the injector located on the lefthand side of the nozzle axis. The highly fluctuating velocity field there is the origin of turbulence fluctuations. They enter the nozzle and have significant impact on the cavitation inception and the subsequent spray disintegration. The pressure plot in Fig. 5 (right) is scaled within a tight range in order to visualize the turbulent pressure fluctuations in the spray domain. In regions where the pressure falls below the saturation pressure, cavitation occurs.

Figure 6 shows the iso-surfaces of the liquid (blue) and vapor (red) volume fraction at certain time instants in a three-dimensional view. After approximately $0.4 \mathrm{~ms}$ the spray reaches a quasi-steady state. During the initial period of injection the liquid forms the wellknown mushroom visible at 0.1 and $0.2 \mathrm{~ms}$ after start of injection. The rim of the mushroom further disintegrates into relatively large ligaments which move with low velocity through the spray domain. Most of them leave the domain or reunite with the liquid core, but some ligaments impinge later on the upper wall and remain there. At $0.2 \mathrm{~ms}$ a fan-shaped liquid core is visible. But owing to its instability, it disintegrates into much smaller ligaments. The red surfaces in the nozzle region at 0.2 and $0.4 \mathrm{~ms}$ indicate cavitation. After approximately 0.4 $\mathrm{ms}$ a developed spray is visible. This means the plots at $0.4,0.85$ and $1 \mathrm{~ms}$ show similarities, although the fully transient behavior of the break-up leads to remarkable differences in the liquid core length, the spray angle and the occurrence of cavitation.
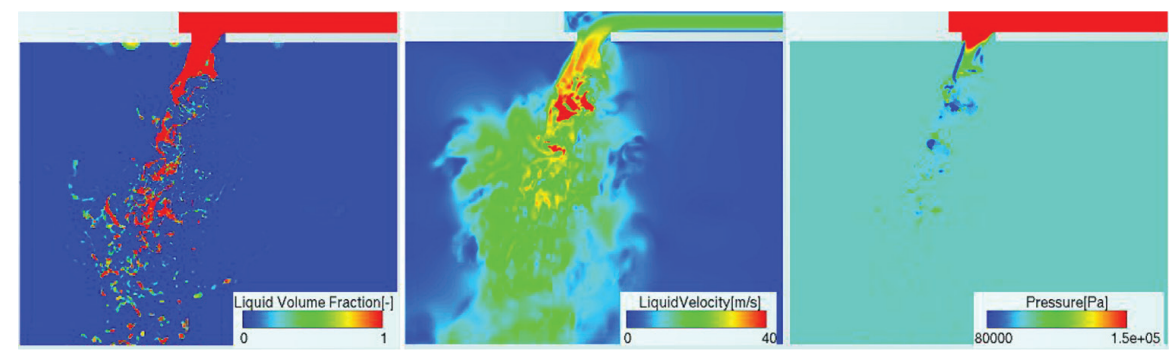

Figure 5: Center cuts through the nozzle showing liquid volume fraction (left), velocity (middle) and pressure (right) at $1 \mathrm{~ms}$. 


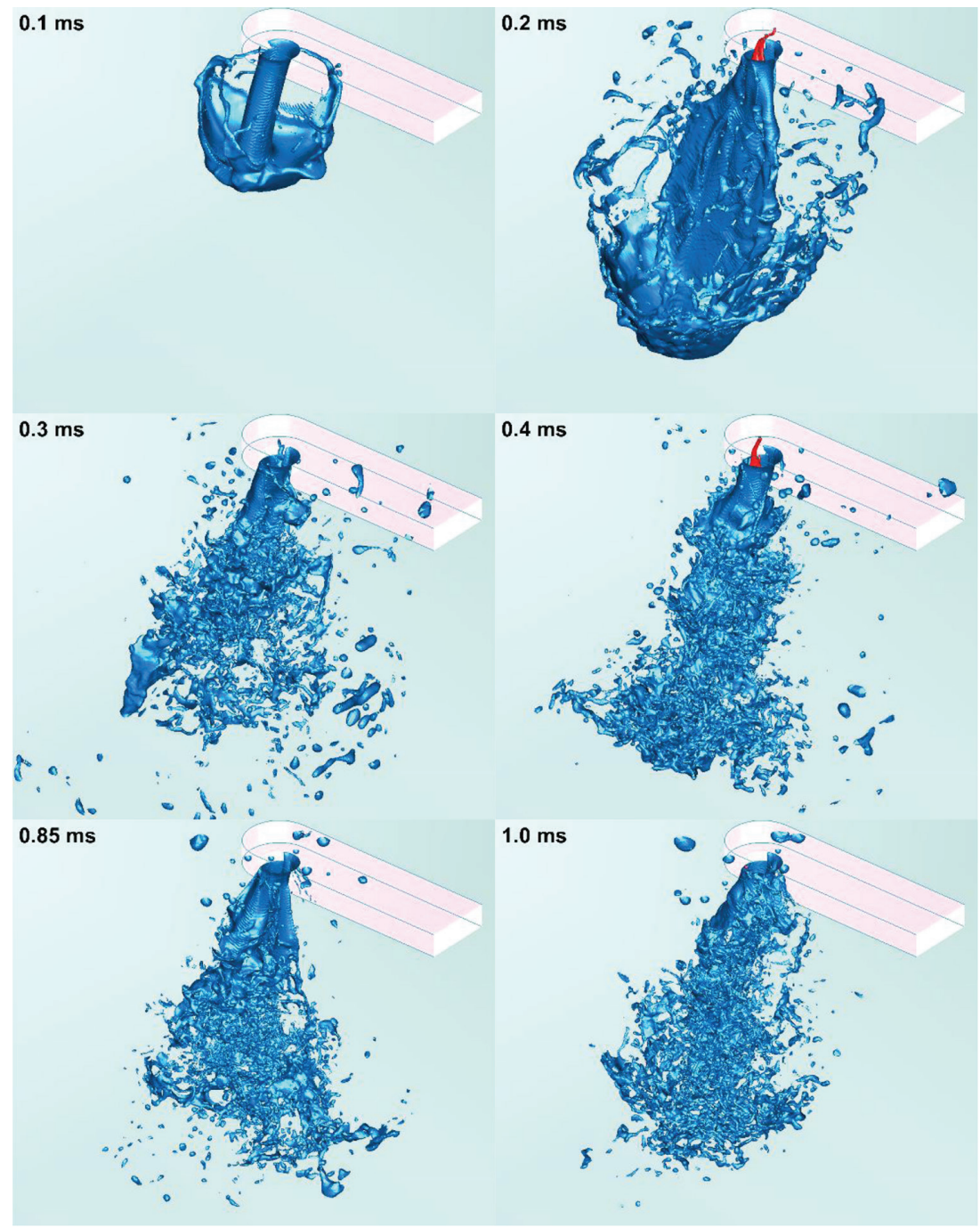

Figure 6: Volume fraction iso-surfaces for liquid (blue, $\alpha_{\text {liq }}=0.5$ ) and vapor (red, $\alpha_{\text {vap }}=0.2$ ) showing the spray evolution at $0.1,0.2,0.3,0.4,0.85$ and $1.0 \mathrm{~ms}$.

Figure 7 gives an insight into the highly unsteady and impressing cavitation pattern inside the nozzle. It shows in a closer view the vapor and liquid iso-surfaces of the volume fraction, velocity vector plots in a cut crossing the nozzle axis at the exit, and streamlines colored by the local liquid velocity. In the early stage of injection, at 0.2 and $0.275 \mathrm{~ms}$, two moving cavitation strings are formed in the center of two vortexes where the local pressure is lower than the saturation pressure. The fully developed cavitation strings prance around the nozzle axis and attach on the upper injector wall. The downstream collapse of the cavitation zone is located outside the nozzle already in the spray domain. At later time instants, only one highly transient cavitation string is visible. Its origin strongly varies along the sharp entrance edge of the nozzle, as it can be seen exemplarily at $0.47 \mathrm{~ms}$ where the origin is located on the 


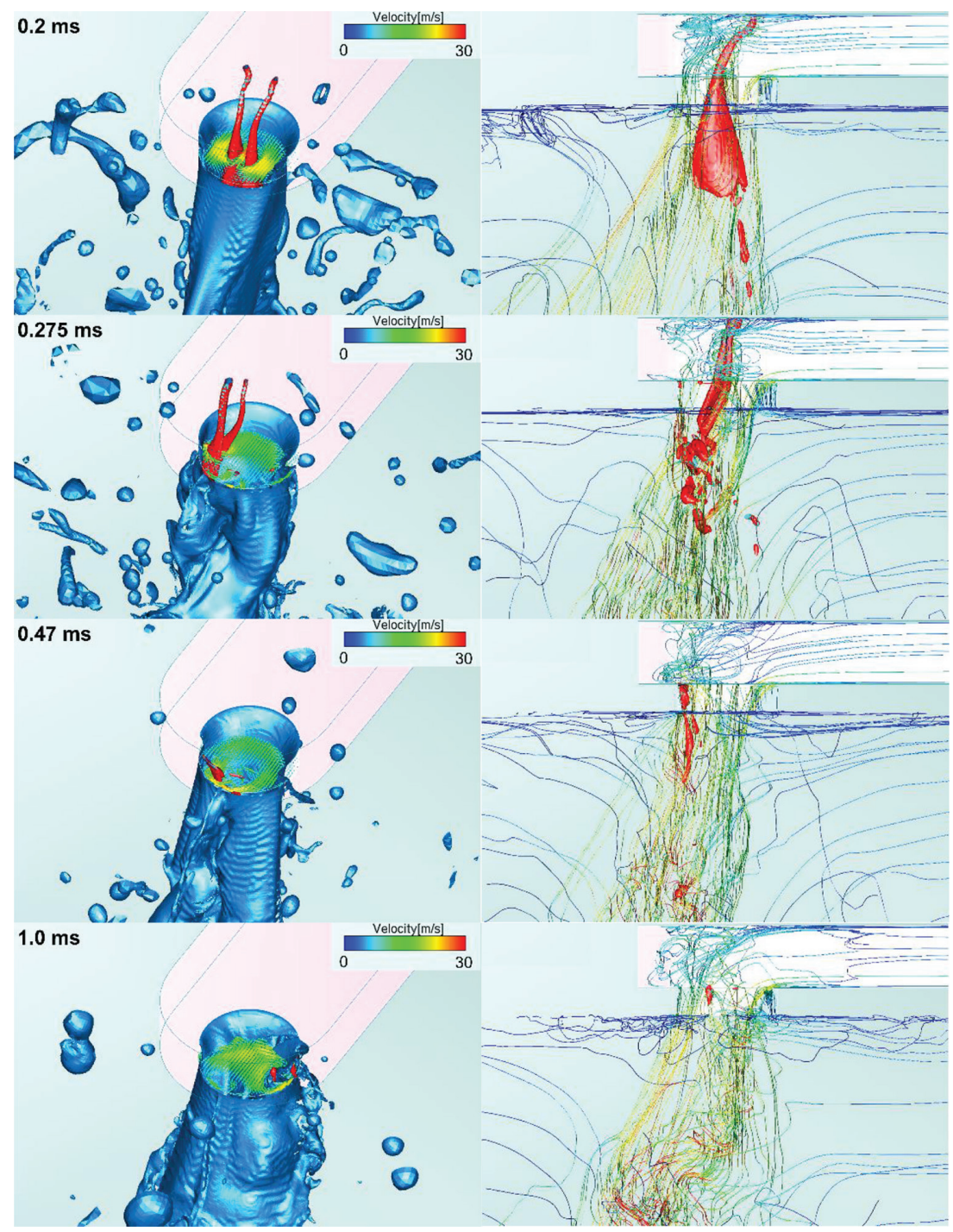

Figure 7: Vapor and liquid volume fraction iso-surfaces $\left(\alpha_{\text {vap }}=0.2, \alpha_{\text {liq }}=0.5\right)$, velocity vectors in a cut crossing the nozzle axis and velocity streamlines showing the string cavitation at $0.2,0.275,0.47$ and $1 \mathrm{~ms}$.

left-hand side of the nozzle axis. From time to time the string cavitation reattaches on the upper injector wall, collapses after a while, and is formed again in a new cycle starting from another, as it seems stochastically chosen, position. The plot at $1 \mathrm{~ms}$ shows exemplarily a time instant with rather weak cavitation. The cavitation is fully driven by the large scale turbulent vortexes generated in the dead volume of the injector. A direct relation between cavitation intensity and instantaneous liquid core length could not been observed. The break-up intensity is rather related to the instantaneous turbulence fluctuations in and also downstream the nozzle. 

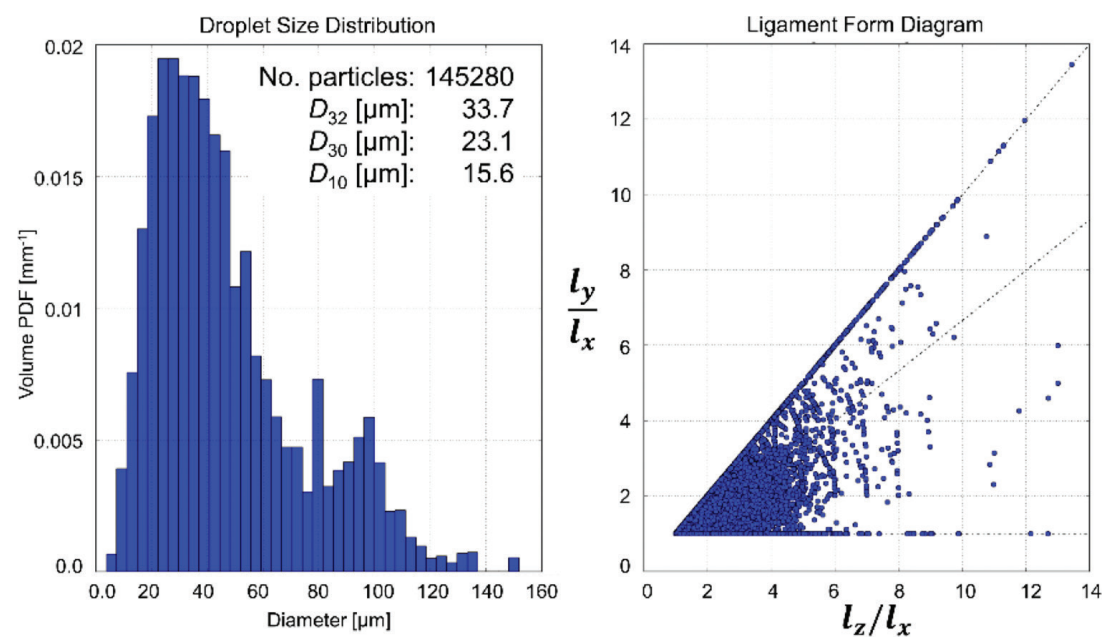

Figure 8: Volume-based droplet size distribution (left) and ligament form diagram (right) for all evaluated ligaments between 0.5 and $1.0 \mathrm{~ms}$.

Finally, Fig. 8 shows the volume based droplet size distribution and the ligament form diagram for all evaluated ligaments in the time period from 0.5 to $1.0 \mathrm{~ms}$ where a quasi-steady spray pattern exists. For demonstration purposes the ligament evaluation has been performed over the whole spray domain and for every $50 \mu \mathrm{s}$. More than 145000 particles have been detected during this evaluation time. The droplet size distribution was calculated with the volume-equivalent sphere diameter of each ligament. The obtained Sauter mean diameter was $33.7 \mu \mathrm{m}$. Since the ligament evaluation was performed over the whole domain and not in a further downstream region where the break-up is already completed, several non-spherical particles created during the initial stage of the break-up can be expected. The form diagram in Fig. 8 right confirms this expectation, since a strongly non-uniform particle shape distribution is visible. The maximum ratios of the particle lengths, $l_{y} / l_{x}$ and $l_{z} / l_{x}$, reach values up to 14 , which indicate thin lamellas or very streaky ligaments. If the ligament detection is performed in an evaluation volume further downstream, one can expect a form diagram with less non-spherical particles and a smaller Sauter mean diameter.

\section{SUMMARY AND CONCLUSIONS}

The aim of this study was to perform a further application test of the recently developed method for three-phase simulations of cavitating injector flow and liquid spray break-up by combination of Volume-of-fluid (VOF) and Eulerian-Eulerian (EE) methods [10]. Furthermore, a method for quantification of non-spherical ligaments was proposed. The test case, reported by [4], consists of a simplified industrial gasoline injector segment with diameter $D=226 \mu \mathrm{m}$. The VOF-LES simulation shows the transient evolution of the spray pattern, and gives insight into the complex injector flow showing unsteady vortex formation and string cavitation. From the simulation results it can be concluded that LES is mandatory for resolving such highly transient flow phenomena contributing to the spray break-up. A direct correlation between cavitation intensity and break-up intensity was not observed. But the disintegration is clearly related to the fluctuation intensity of the turbulent eddies. For each individual ligament the extensions along their principal axes of inertia are determined, and the length ratios are inscribed into the so-called form diagram. This is a useful illustration for assessing the amount of non-spherical particles, 
and consequently the completeness of the break-up. Comparing the cavitating three-phase simulation with a non-cavitating two-phase simulation, enhancing the workflow for nonisothermal phases, and performing further test cases with measured droplet size distribution are part of future investigations.

\section{REFERENCES}

[1] Menard, T., Tanguy, S. \& Berlemont A., Coupling level set/VOF/ghost fluid methods: validation and application to 3D simulation of the primary break-up of a liquid jet. International Journal of Multiphase Flow, 33, pp. 510-524, 2007. https://doi.org/10.1016/j.ijmultiphaseflow.2006.11.001

[2] Shinjo, J. \& Umemura, A., Simulation of liquid jet primary breakup: Dynamics of ligament and droplet formation. International Journal of Multiphase Flow, 36(7), pp. 513-532, 2010. https://doi.org/10.1016/j.ijmultiphaseflow.2010.03.008

[3] Hermann, M., Detailed numerical simulations of the primary atomization of a turbulent liquid jet in crossflow. Journal of Engineering for Gas Turbines and Power, 132(6), 2010. https://doi.org/10.1115/1.4000148

[4] Ishimoto, J., Sato, F. \& Sato, G., Computational prediction of the effect of microcavitation on an atomization mechanism in a gasoline injector nozzle. Journal of Engineering for Gas Turbines and Power, 132(6), 2010. https://doi.org/10.1115/1.4000264

[5] Schmidt, D.P., Ruland, C.J. \& Corradini, M.L., A fully compressible model of small, high speed cavitating nozzle flows. Atomization and Sprays, 9, pp. 255-276, 1999. https://doi.org/10.1615/atomizspr.v9.i3.20

[6] Lu, N.X., Demoulin, F.X., Reveillon, J. \& Chesnel, J., Large Eddy simulation of a cavitating multiphase flow for liquid injection. Proceeding of : ILASS, Bremen, Germany, 2014.

[7] Sauer, J., Winkler, G. \& Schnerr, G. H., Cavitation and condensation - common aspects of physical modeling and numerical approach. Chemical Engineering \& Technology, 23, pp. 663-666, 2000.

[8] Cailloux, M., Helie, J., Reveillon, J. \& Demoulin, F.X., Large Eddy simulation of a cavitating multiphase flow with OpenFoam. Journal of Physics: Conference Series, 656(1), 2015. https://doi.org/10.1088/1742-6596/656/1/012081

[9] Yu, H., Goldsworthy, L, Brandner, P.A. \& Garanijy, V., Development of a compressible multiphase cavitation approach for diesel spray modelling. Applied Mathematical Modelling, 45, pp. 705-727, 2017. https://doi.org/10.1016/j.apm.2017.01.035

[10] Edelbauer, W., Numerical simulation of cavitating injector flow and liquid spray break-up by combination of Eulerian-Eulerian and volume-of-fluid methods. Computers and Fluids, 144, pp. 19-33, 2017. https://doi.org/10.1016/j.compfluid.2016.11.019

[11] Sou, A., Bicer, B. \& Tomiyama, A., Numerical simulation of incipient cavitation flow in a nozzle of fuel injector. Computers \& Fluids, 103, pp. 42-48, 2014.

https://doi.org/10.1016/j.compfluid.2014.07.011 
[12] Biçer, B. \& Sou, A., Application of the improved cavitation model to turbulent cavitating flow in fuel injector nozzle. Applied Mathematical Modelling, 40(7-8), pp. 4712-4726, 2015. https://doi.org/10.1016/j.apm.2015.11.049

[13] AVL FIRE ${ }^{\mathrm{TM}}$, user manual of version v2017, 2017.

[14] Alajbegovic, A., Greif, D., Basara, B. \& Iben, U., Cavitation calculation with the twofluid model. Proceeding of 3rd European-Japanese Two-Phase Flow Group Meeting, Italy, 2003.

[15] Greif, D. \& Srinivasan, V., Numerical prediction of erosive cavitating flows in injection equipment, SAE 2011-24-0004, 2011.

[16] Greif, D., Sampl, P. \& Edelbauer, W., Cavitating injector flow simulations considering longitudinal and lateral needle displacement. International Journal of Automotive Engineering, 5, pp. 85-90, 2014.

https://doi.org/10.20485/jsaeijae.5.2_85

[17] Kobayashi, H., The subgrid-scale models based on coherent structures for rotating homogeneous turbulence and turbulent channel flow. Physics of Fluids, 17, 2005. https://doi.org/10.1063/1.1874212

[18] Kobayashi, H., Hama, F. \& Wu, X., Application of a local SGS model based on coherent structures to complex geometries. International Journal of Heat and Fluid Flow, 29, pp. 640-653, 2008. https://doi.org/10.1016/j.ijheatfluidflow.2008.02.008

[19] Ubbink, O. \& Issa, R.I., A method for capturing sharp fluid interfaces on arbitrary meshes. Journal of Computational Physics, 153, pp. 26-50, 1999.

https://doi.org/10.1006/jcph.1999.6276

[20] Walz, K., Die Betonstraße, 11, pp. 27-36, 1936 (German).

[21] Gaskell, P.H. \& Lau, A.K.C., Curvature-compensated convective Transport: SMART, a new boundedness-preserving transport algorithm. International Journal for Numerical Methods in Fluids, 8, pp. 617-641, 1988. https://doi.org/10.1002/fld.1650080602

[22] Patankar, S.V. \& Spalding, D.B., A calculation procedure for heat, mass and momentum transfer in three-dimensional parabolic flows. International Journal of Heat and Mass Transfer, 15(10), pp. 1510-1520, 1972.

[23] Ferziger, J.H. \& Peric M., Computational methods for fluid dynamics, Springer, New York, 1996. 